\title{
Investigating class rarity in big data
}

\author{
Tawfig Hasanin, Taghi M. Khoshgoftaar, Joffrey L. Leevy ${ }^{*}$ and Richard A. Bauder
}

*Correspondence: jleevy2017@fau.edu Florida Atlantic University, 777 Glades Road, Boca Raton 33431, FL, USA

\begin{abstract}
In Machine Learning, if one class has a significantly larger number of instances (majority) than the other (minority), this condition is defined as class imbalance. With regard to datasets, class imbalance can bias the predictive capabilities of Machine Learning algorithms towards the majority (negative) class, and in situations where false negatives incur a greater penalty than false positives, this imbalance may lead to adverse consequences. Our paper incorporates two case studies, each utilizing a unique approach of three learners (gradient-boosted trees, logistic regression, random forest) and three performance metrics (Area Under the Receiver Operating Characteristic Curve, Area Under the Precision-Recall Curve, Geometric Mean) to investigate class rarity in big data. Class rarity, a notably extreme degree of class imbalance, was effected in our experiments by randomly removing minority (positive) instances to artificially generate eight subsets of gradually decreasing positive class instances. All model evaluations were performed through Cross-Validation. In the first case study, which uses a Medicare Part B dataset, performance scores for the learners generally improve with the Area Under the Receiver Operating Characteristic Curve metric as the rarity level decreases, while corresponding scores with the Area Under the Precision-Recall Curve and Geometric Mean metrics show no improvement. In the second case study, which uses a dataset built from Distributed Denial of Service attack attack data (POSTSlowloris Combined), the Area Under the Receiver Operating Characteristic Curve metric produces very high-performance scores for the learners, with all subsets of positive class instances. For the second study, scores for the learners generally improve with the Area Under the Precision-Recall Curve and Geometric Mean metrics as the rarity level decreases. Overall, with regard to both case studies, the Gradient-Boosted Trees (GBT) learner performs the best.
\end{abstract}

Keywords: Big data, Class imbalance, Machine learning, Medicare fraud, POSTSlowloris, Class rarity, Undersampling

\section{Introduction}

When called upon to define big data, researchers and practitioners in the field of data science frequently refer to the six V's: volume, variety, velocity, variability, value, and veracity [1]. Volume, most certainly the best-known property of big data, is associated with the profusion of data produced by an organization. Variety covers the handling of structured, unstructured, and semi-structured data. Velocity takes into account how quickly data is manufactured, issued, and dealt with. Variability refers to the fluctuations in data. Value is often regarded as a critical attribute because it is required for effective decision-making. Veracity is associated with the fidelity of data.

(c) The Author(s) 2020. This article is licensed under a Creative Commons Attribution 4.0 International License, which permits use, sharing adaptation, distribution and reproduction in any medium or format, as long as you give appropriate credit to the original author(s) and the source, provide a link to the Creative Commons licence, and indicate if changes were made. The images or other third party material in this article are included in the article's Creative Commons licence, unless indicated otherwise in a credit line to the material. If material is not included in the article's Creative Commons licence and your intended use is not permitted by statutory regulation or exceeds the permitted use, you will need to obtain permission directly from the copyright holder. To view a copy of this licence, visit http://creativeco mmons.org/licenses/by/4.0/. 
A definition of big data related to a minimum number of dataset instances has not been established in the literature. For example, in [2] this minimum was identified as 100,000 instances, but other works use 1,000,000 instances [3, 4]. The increasing reliance on big data applications is pushing the development of efficient knowledge-extraction methods for this type of data.

Any dataset containing majority and minority classes, e.g., normal transactions and fraudulent transactions for a large bank over the course of a day, can be viewed as classimbalanced. Various degrees of class imbalance exist, ranging from slightly imbalanced to rarity. Class rarity in a dataset is defined by comparatively inconsequential numbers of positive instances [5], e.g., the occurrence of 10 fraudulent transactions out of 1,000,000 total transactions generated daily for a bank. Binary classification is usually associated with class imbalance since many multi-class classification problems can be managed by breaking down the data into multiple binary classification tasks. The minority (positive) class, which comprises a smaller part of the dataset, is usually the class of interest in real-world problems [2], as opposed to the majority (negative) class, which comprises the larger part of the dataset. Although class imbalance affects both big and non-big data, the adverse effects are usually more perceptible in the former, due to the existence of extreme degrees of class imbalance within big data [6] as a result of voluminous overrepresentation of the negative (majority) class within datasets.

Machine Learning $(M L)$ algorithms are usually better classifiers than traditional statistical techniques [7-9], but these algorithms cannot properly differentiate between majority and minority classes if the dataset is plagued by class rarity. The inability to sufficiently distinguish majority from minority classes is analogous to searching for the proverbial needle in a haystack and could result in the classifier labeling almost all instances as the majority (negative) class. Performance metric values based on such poor analysis would be deceptively high. When the occurrence of a false negative incurs a higher cost than a false positive, a classifier's bias towards the majority class may lead to adverse consequences [10]. As an example, if defective flight-control software for a jetliner is labeled as defect-free (false negative), the end result of proceeding with the production of this software could be catastrophic. On the other hand, if the software is free of defects but was mislabeled as faulty, the outcome would most certainly not be life-threatening.

Our work evaluates the classification performance of three learners (Gradient-Boosted Trees, Logistic Regression, Random Forest) with three performance metrics (Area Under the Receiver Operating Characteristic (ROC) Curve, Area Under the Precision-Recall Curve, Geometric Mean), and also compares results from two case studies involving imbalanced big data from different application domains. Class rarity was injected by randomly removing positive instances to artificially generate eight subsets of positive class instances $(1,000,750,500,400,300,200,100$, and 50). For comparative purposes, we also evaluated the original positive class instances for both datasets. All model evaluations were performed through Cross- Validation. For our processing needs, we use the Apache Spark [11] and Apache Hadoop frameworks [12-14], both of which can handle big data.

The first case study is based on the Medicare Part B dataset $[15,16]$. The original dataset contains $1,409(0.038 \%)$ positive class instances out of 3,692,555 total instances. The results indicate that performance scores for the learners generally improve with the Area 
Under the ROC Curve metric as the rarity level decreases, while corresponding scores with the Area Under the Precision-Recall Curve (AUPRC) and Geometric Mean (GM) metrics remain relatively unchanged. The second case study is based on a combination of two datasets: POST dataset [17] and Slowloris dataset [18]. POST and Slowloris are two types of Distributed Denial of Service (DDoS) attacks. The resultant dataset, referred to as POSTSlowloris Combined, contains 6,646 (0.203\%) positive instances out of 3,276,866 total instances. The results show that the Area Under the ROC Curve (AUC) metric yields very high-performance scores for the learners, with all subsets of positive class instances. With the exception of the GM metric score obtained by the Random Forest (RF) learner in the second study, scores for the learners generally improve with the AUPRC and GM metrics as the rarity level decreases. For both case studies, our results show that the GBT learner produces the best overall performance.

To the best of our knowledge, our work is unique in investigating class rarity in big data through the use of three learners and three performance metrics. We also demonstrate the effectiveness and versatility of our approach by using case studies with imbalanced big data from different application domains.

The remainder of this paper is organized as follows: Section "Related Work" provides an overview of literature that focuses on datasets with class rarity; Section "Case studies datasets" provides details on the Medicare Part B and POSTSlowloris Combined datasets; Section "Methodologies" describes the different aspects of the methodology used to develop and implement our approach, including injection of rarity, model evaluation, and experiment design; Section "Results and discussion" presents and discusses our empirical results; Section "Conclusion" concludes our paper with a summary of the work presented and suggestions for related future work.

\section{Related work}

Our search for related work found many more big data studies involving severe class imbalance than class rarity. It should be noted that research on class rarity is still in its infancy.

In [11], researchers examine Evolutionary Undersampling (EUS) in cases of class imbalance in big data, based on the initial knowledge that EUS had shown promise in addressing class imbalance in traditional data. The EUS approach is implemented within the Apache Spark framework, and compared with their previous implementation of EUS with the Apache Hadoop framework. The base learner in both implementations is the C4.5 decision tree learner which is incorporated into the overall class balancing and classification process. EUS provides a fitness function for a prototype selection method, where the fitness function aims to find the proper balance between reduction (undersampling) of training data instances and classification performance [19]. The authors recognized that divide-and-conquer methods based on MapReduce can potentially be affected by a lack of density from the minority class in the subsets created. As a result, the in-memory operation of Apache Spark is modified such that the majority and minority class instances can be independently managed. This enables a relatively higher number of minority class instances to be retained in each subset created. The case study data was comprised of variants of two big data sets, i.e., ECDBL'14 and KDD Cup 1999 datasets. Two subsets, $50 \%$ and 25\%, of the ECBDL'14 data were used, each with 631 features 
and a class ratio of 98:2. Three variants of the KDD Cup 1999 data were used, based on different binary class combinations, each with 41 features and the approximate class ratio values of $95: 1,3450: 1$, and 74,680:1 for the three datasets. The dataset with the ratio of 74,680:1 definitely has class rarity, with 52 positive instances and 3,883,370 negative instances. The key results observed in the paper include: Apache Spark framework had shorter runtimes than Apache Hadoop; EUS performed better than Random Undersampling (RUS), but as expected, its runtime was much longer than that of RUS. The best overall values of GM classification performance metric for EUS and RUS were 0.672 and 0.662 , respectively. The focus of this work, however, is not on class rarity.

An evaluation of the performance of several methods used to address class imbalance in big data was performed in [20], where all methods were implemented within the Apache Hadoop framework, with RF as the base classifier. These methods included Random Oversampling (ROS), RUS, Synthetic Minority Over-sampling TEchnique (SMOTE), and a cost-sensitive learning version of RF. The datasets in this study ranged from approximately 435,000 to 5,700,000 instances, with feature set sizes between 2 and 41 . Majority-to-minority class ratios varied between $80: 20$ and 77,670:1. There were several clear instances of big data datasets with class rarity in this work: (52 positive instances and 3,883,370 negative instances; 52 positive instances and 972,781 negative instances; 15 positive instances and 1,165,011 negative instances; 20 positive instances and 1,553,348 instances; 26 positive instances and 1,941,685 negative instances; and 52 positive instances and 3,883,370 negative ones. The results of the experiment were inconclusive, as there was no best model among these four diverse algorithms. The authors state that the best performing algorithm depends on the number of mappers with MapReduce that are chosen to run the experiment. For GM, the best overall values of ROS, RUS, SMOTE, and RF were 0.986, 0.984, 0.914, and 0.965, respectively. Just like the related work in the previous paragraph, the focus of this related study is not on class rarity.

The work in [21] examined a modification to DeepQA, the technology that powered IBM Watson on the Jeopardy! game show. DeepQA is a question-and-answer, natural language processing system that can help professionals make critical and timely decisions [22]. During the process of gamification, a significant majority of generated question labels are incorrect because of missing data, badly constructed training questions, incorrect feature merging, answer key errors, or missing domain features. The massive number of false to true labels creates the class imbalance condition. The authors combined a data-Level approach (manual question and answer vetting, over-sampling) with an algorithm-Level approach (Logistic Regression (LR) with a regularization term) to address the issue of high-class imbalance. The dataset in this study contained approximately 720,000 instances and 400 features, with a majority-to-minority class ratio of 6930:1. This means that there are about 100 positive instances and 719,900 negative instances. The results show that regularized LR with over-sampling outperformed unregularized LR with over-sampling in terms of accuracy, which increased from 1.6 to $28 \%$. It is worth mentioning that for this study, some data scientists may not consider the total number of dataset instances $(720,000)$ as big data.

Finally, in [5], the impact of class rarity on big data is evaluated. The researchers use publicly available Medicare data and map known fraudulent providers, from the List of Excluded Individuals/Entities (LEIE) [23], as labels for the positive class. This Medicare 
dataset contains 3,691,146 instances, with 1,409 (0.038\%) positive class instances. Three learners are used to evaluate fraud detection performance. Additional datasets are artificially generated from the original Medicare dataset by gradually decreasing the number of positive class instances (while leaving the number of negative class instances untouched). The generated datasets have positive class counts as low as 50 . The researchers observed that the original Medicare dataset generally performs better than the datasets with small counts of positive instances. A noticeable deterioration in performance occurs at 200 positive instances and lower count values. Based on the results of the experiment, the researchers proposed a taxonomy of class imbalance: positive counts of 1409 and 1000 (imbalanced or highly imbalanced); positive counts of 750, 500, 400 and 300 (severely imbalanced); positive counts of 200, 100, and 50 (class rarity). While this study focuses on class rarity, the approach is based on a dataset from one application domain. In our work, we attempt to generalize our approach by using datasets from two different application domains. Moreover, we use three performance metrics (AUC, AUPRC, GM), whereas this related study only uses AUC.

\section{Case studies datasets}

Our work includes two case studies. The dataset used in the first case study came from a different application domain than the dataset used in the second case study. The first case study is based on the Medicare Part B dataset, which contains 1,409 (0.038\%) positive class instances out of 3,692,555 total instances. The second case study is based on a combination of two datasets: POST dataset and Slowloris dataset. The merged dataset, referred to as POSTSlowloris Combined, contains 6,646 (0.203\%) positive instances out of 3,276,866 total instances.

\section{Medicare Part B}

The Medicare Physician and Other Supplier (Part B) dataset used in this paper spans years 2012 to 2016. It is provided by the Centers for Medicare and Medicaid Services. The Part B dataset, which includes claims information for each procedure that a physician/provider performs in a given year, is derived from administrative claims data for Medicare beneficiaries enrolled in the Fee-For-Service program, where all claims information is recorded after payments are made [15]. Therefore, we can safely assume that these datasets are already reasonably cleansed.

\section{POSTSlowloris}

DDoS attacks are carried out through various methods designed to deny network availability to legitimate users [24]. Hypertext Transfer Protocol (HTTP) contains several exploitable vulnerabilities and is often targeted for DDoS attacks $[25,26]$. During a Slow HTTP POST attack, legitimate HTTP headers are sent to a target server. The message body of the exploit must be the correct size for communication between the attacker and the server to continue. Communication between the two hosts becomes a drawnout process as the attacker sends messages that are relatively very small, tying up server resources. This effect is worsened if several POST transmissions are done in parallel. During a Slowloris attack, numerous HTTP connections are kept engaged for as long 
as possible. Only partial requests are sent to a web server, and since these requests are never completed the available connections for legitimate users becomes zero.

Data collection for the POST and Slowloris DDoS attacks was performed within a real-world network setting. An ad hoc Apache web server, which was set up within a campus network environment, served as a viable target. The Switchblade 4 tool from the Open Web Application Security Project and a Slowloris.py attack script [27] were used to generate attack packets for POST and Slowloris, respectively. Attacks were launched from a single host computer in hourly intervals. Attack configuration settings, such as connection intervals and number of parallel connections, were varied, but the same PHP form element on the web server was targeted during the attack.

\section{Methodologies}

This section is a report on the methodologies followed, including our reasons for choosing them. We discuss the big data processing framework, injection of rarity, one-hot encoding, classifiers, performance metrics, model evaluation, addressing of randomness, and experiment design.

\section{Big data processing framework}

To facilitate the use of ML in big data analytics, data engineers build algorithms within software modules or packages, ensuring that reliability, speed, and scale are factored in. For the ML tasks, we use a state-of-the-art library, Machine Learning Library (MLlib), provided by Apache Spark [28, 29], hereinafter referred to as Spark. Compared to traditional ML methods, Spark is exponentially faster at data processing, and is one of the largest open source projects for big data processing [30]. In addition, we utilize Apache Hadoop [12-14], which provides Hadoop Distributed File System (HDFS), a scalable component capable of storing large files across node clusters, and also utilize Yet Another Resource Negotiator (YARN) [31], a component used for job management and scheduling in High Performance Computing (HPC).

For performance stability, we kept our data partitions invariant and memory use fixed during the experiments. Thus, the number of distributed data partitions and the number of the cluster slave nodes were picked based on the available resources of our HPC cluster.

\section{Injecting rarity}

The Medicare Part B dataset contains $1,409(0.038 \%)$ positive class instances out of 3,692,555 total instances, and the POSTSlowloris Combined dataset contains 6,646 $(0.203 \%)$ positive instances out of $3,276,866$ total instances.

From this original data, we artificially generate eight subsets with gradually decreasing numbers of positive class instances $(1,000,750,500,400,300,200,100$, and 50). To create each subset, we built the model with the same number of negative instances and randomly picked a basket of positive counts from the positive instances. Table 1, which provides information on the minority (positive) and majority (negative) classes, summarizes the datasets (subsets) used in our experiment. 
Table 1 Summary of datasets

\begin{tabular}{lllll}
\hline Positives & Negatives & Total & \%Positives & \%Negatives \\
\hline a. Medicare Part B & & & & \\
1,409 (all) & $3,691,146$ & $3,692,555$ & 0.038 & 99.962 \\
1,000 & $3,691,146$ & $3,692,146$ & 0.027 & 99.973 \\
750 & $3,691,146$ & $3,691,896$ & 0.020 & 99.980 \\
500 & $3,691,146$ & $3,691,646$ & 0.014 & 99.986 \\
400 & $3,691,146$ & $3,691,546$ & 0.011 & 99.989 \\
300 & $3,691,146$ & $3,691,446$ & 0.008 & 99.992 \\
200 & $3,691,146$ & $3,691,346$ & 0.005 & 99.995 \\
100 & $3,691,146$ & $3,691,246$ & 0.003 & 99.997 \\
50 & $3,691,146$ & $3,691,196$ & 0.001 & 99.999 \\
b. PostSlowloris Combined & & & 99.797 \\
6,646 (all) & $3,270,220$ & $3,276,866$ & 0.203 & 99.969 \\
1,000 & $3,270,220$ & $3,271,220$ & 0.031 & 99.977 \\
750 & $3,270,220$ & $3,270,970$ & 0.023 & 99.985 \\
500 & $3,270,220$ & $3,270,720$ & 0.015 & 99.988 \\
400 & $3,270,220$ & $3,270,620$ & 0.012 & 99.991 \\
300 & $3,270,220$ & $3,270,520$ & 0.009 & 99.997 \\
200 & $3,270,220$ & $3,270,420$ & 0.006 & 99.998 \\
100 & $3,270,220$ & $3,270,320$ & 0.003 & \\
50 & $3,270,220$ & $3,270,270$ & 0.002 & \\
\hline
\end{tabular}

\section{One-hot encoding}

Another factor needing attention is categorical features. In their raw form, these are generally not compatible with ML algorithms. Additionally, if the categorical features were indexed, some models assume that there is a logical order or a value of the indices. Such subsets of categorical features are known as ordinal features. A nominal feature, unlike an ordinal one, is a categorical feature whose instances can take a value that cannot be organized in a logical sequence [7]. In our work, all categorical features (nominal) were transformed into dummy variables using a one-hot encoding method [32], allowing conversion of nominal features into numerical values. One disadvantage of this method is that a new number of features equaling $C-1$ is generated from each feature, where $C$ is the number of categories belonging to the specific feature, and consequently, the total feature space increases in size.

\section{Classifiers}

Our work uses three off-the-shelf learners (RF, GBT, LR), all of which are available in the Spark MLlib. These classifiers were selected to provide good coverage of various ML model families. Performance-wise, the three classifiers are regarded favorably, and they incorporate both ensemble and non-ensemble algorithms, providing a reasonable breadth of fraud detection results for assessing the impact of rarity in Big Data [33, 34]. In this section, we describe each model and note configuration and hyperparameter changes that differ from the default settings. 
- RF is a regression and classification model that employs an ensemble learning approach. RF constructs a large number of independent decision trees during training and returns a final model prediction that is the average or majority vote of the individual tree results. We build each RF learner with 100 trees. The parameter that caches node IDs for each instance was set to true, and the maximum memory parameter was set to $1024 \mathrm{MB}$ in order to minimize training time. The maximum bins parameter, which is for discretizing continuous features, was set to 2 since we use one-hot encoding on categorical variables.

- GBT is a boosting ensemble method, but unlike RF, new models are added to the ensemble sequentially. The predicted values are evaluated with the actual values, allowing the algorithm to pinpoint and correct previously mislabeled instances. The parameter that caches node IDs for each instance was set to true, and the maximum memory parameter was set to $1024 \mathrm{MB}$ to minimize training time.

- LR is similar to linear regression except that it employs a sigmoidal (logistic) function to generate class probabilities to predict class membership. The bound matrix was set to match the shape of the data (number of classes and features) so that the algorithm knows the number of classes and features the dataset contains. The bound vector size was equal to 1 for binomial regression and no thresholds were set for binary classification.

\section{Performance metrics}

Accuracy or error rate is usually derived from a naive 0.50 threshold that is used in the prediction of one out of the two classes. This is usually impractical since in most realworld situations the two classes are imbalanced, creating the majority and minority class groups. The Confusion Matrix (CM) for a binary classification problem is shown in Table 2 [10], where positive, the class of interest, is the minority class and negative is the majority class.

- True positive (TP) are positive instances correctly identified as positive.

- True negative (TN) are negative instances correctly identified as negative.

- False positive (FP), also known as Type I error, are negative instances incorrectly identified as positive.

- False negative (FN), also known as Type II error, are positive instances incorrectly identified as negative.

Table 2 Confusion matrix

\begin{tabular}{|c|c|c|c|}
\hline & & \multicolumn{2}{|l|}{ Predicted class } \\
\hline & & Positive & Negative \\
\hline \multirow[t]{2}{*}{ Actual class } & Positive & True positive (TP) & $\begin{array}{l}\text { False negative (FN) (Type } \\
\text { II error) }\end{array}$ \\
\hline & Negative & $\begin{array}{l}\text { False positive (FP) (Type } \\
\text { I error) }\end{array}$ & True negative (TN) \\
\hline
\end{tabular}


From those four broad CM metrics, we may calculate other performance metrics that consider the rates between the positive and the negative class as follows.

- True positive Rate ( $\mathrm{TP}_{\text {rate }}$ ), also known as Recall or Sensitivity, is equal to TP / (TP + $\mathrm{FN})$.

- True negative Rate $\left(\mathrm{TN}_{\text {rate }}\right)$, also known as Specificity, is equal to TN / ( TN + FP ).

- False positive Rate ( $\mathrm{FP}_{\text {rate }}$ ), also known as false alarm rate, is equal to $\mathrm{FP} /(\mathrm{FP}+\mathrm{TN})$, which usually refers to the expectancy of the false positive ratio.

- Positive Predictive Value (PPV), also known as Precision, is equal to TP / ( TP + FP ).

From a comparative standpoint, our work benefits from the use of three performance metrics. The three metrics are commonly used within the ML community for addressing high class imbalance, and are calculated as follows:

- GM, which indicates how well the model performs at the threshold where $\mathrm{TP}_{\text {rate }}$ and $\mathrm{TN}_{\text {rate }}$ are equal, is given by $\sqrt{\mathrm{TP}_{\text {rate }} \times \mathrm{TN}_{\text {rate }}}$.

- AUC calculates the area under the ROC curve, which is a plot of $\mathrm{TP}_{\text {rate }}$ versus $\mathrm{FP}_{\text {rate }}$ at different classification cut-offs.

- AUPRC calculates the area under the Precision-Recall curve. This metric summarizes the trade-off between the PPV and the $\mathrm{TP}_{\text {rate }}$ for a predictive model using different probability thresholds. It is important to note that AUPRC does not account for the number of true negatives.

Note that ROC curves are usually used when there are roughly equal numbers of instances for each class, in other words when the data is balanced [35]. On the other hand, the use of Precision-Recall curves is preferred when there is a moderate to large class imbalance [35]. Since our datasets are severely class-imbalanced, the use of AUC in this study is for comparative purposes only.

\section{Model evaluation}

In ML, one of the commonly used model evaluation methods is Cross-Validation (CV), in which a portion of the data trains the model while the remaining portion validates the built model. Also known as rotation estimation, $k$-fold $\mathrm{CV}$ evaluates predictive models by partitioning the original sample into several folds of approximately equal size. The inducer is trained and tested $k$ times, where each time it is trained on $k-1$ folds and tested on the remaining fold. This is to ensure that all data are used in the classification.

With imbalanced data, one typically uses stratified $k$-fold $C V$, where the minority and majority classes have representative proportions, in each fold, of the class labels from the training data. When compared to regular cross validation, the stratification scheme is generally better suited for addressing bias and variance [36].

Spark and its libraries currently do not offer a CV evaluation method. Thus, for all model evaluations, we implemented our own scalable, stratified $k$-fold CV within Spark. Fig. 1 shows the process flow for our implementation of 5 -fold CV.

Our main reason for using $k$-fold $\mathrm{CV}$ instead of the traditional train versus test method is to ensure that all data are used in the model building/evaluation process. This 


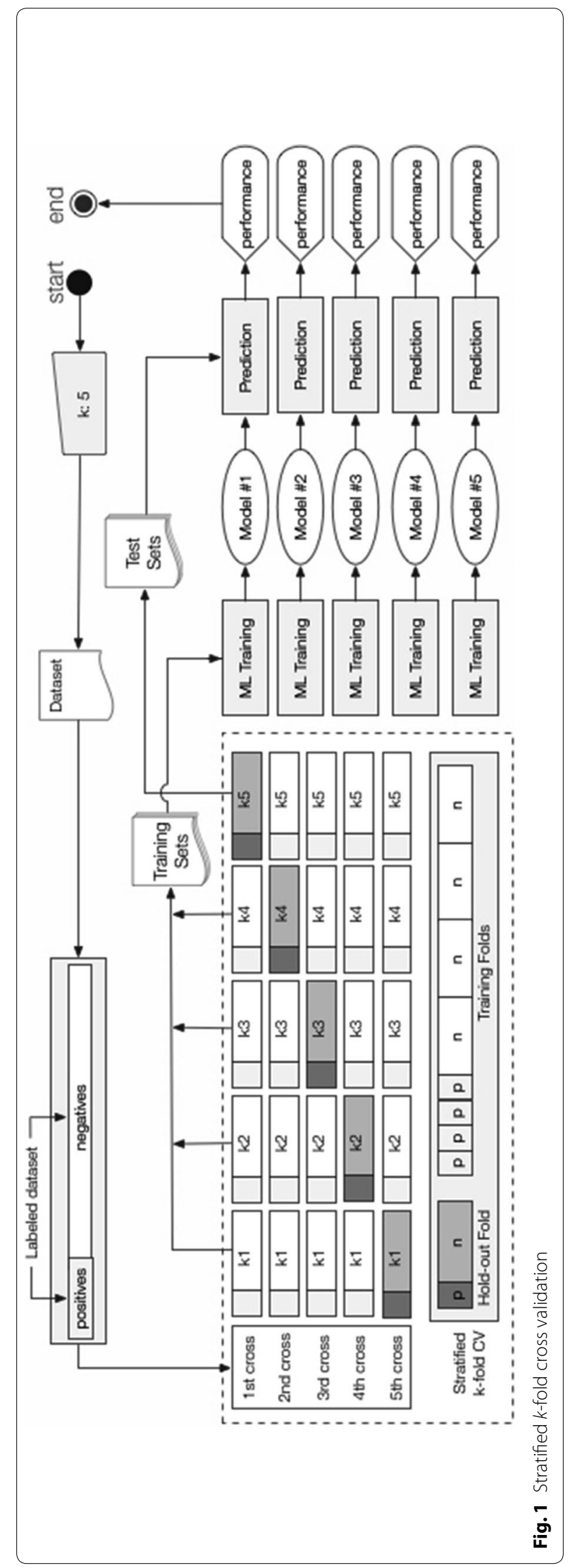


consideration is particularly applicable to our study, which investigates a limited number of positive class instances.

\section{Addressing randomness}

Due to the random generation of the datasets (through random CV splits, the selection of random positive instances, and the random ordering of instances and features prior to model training), the datasets used to build and train the models may retain both good and/or poor instances. Moreover, learners such as RF and GBT may randomly select instances during the construction of each tree. Such randomness may affect final model performance. To address this problem, we use a repetitive models strategy [37] in our work, with each model repeated 10 times.

\section{Experiment design}

The following procedure summarizes the algorithmic steps used in our proposed approach.

1. Distribute the data among HDFS.

2. Perform one-hot encoding: The categorical features space is indexed and one-hot encoded to dummy variables in order to exclude any assumed ordering, by the learner, between the categories of the nominal features. This produces new features equal to one less than the number of categories in each feature.

3. Inject rarity by artificially generating eight subsets with gradually decreasing numbers of positive class instances $(1,000,750,500,400,300,200,100$, and 50). Additionally, we include the original datasets containing all instances of the positive class.

4. Perform 5-fold CV and randomly order the instances and feature space on all datasets. Perform the 5-fold CV with each of the three classifiers (RF, GBT, LR), and evaluate each model produced with the three performance metrics (AUC, AUPRC, GM).

5. Starting from step 3, repeat the entire process 10 times.

To sum things up, we assessed the performance of 2,700 models $(2$ datasets $\times 5$-fold CV $\times$ 3learners $\times 9$ positive counts (rarity) $\times 10$ repetitions). In total, we obtained 8100 performance values for the three performance metrics involved.

\section{Results and discussion}

Tables 3 and 4 show the average results for the Medicare Part B and POSTSlowloris case studies, respectively. Three performance metrics are used in this work (AUC, AUPRC, GM). The classification performance of three learners (GBT, LR, RF) is evaluated with each of the three metrics. The highest score for each row, where a row represents a given learner for a specified metric, is shown in italics.

We artificially generated eight subsets with gradually decreasing numbers of positive class instances $(1,000,750,500,400,300,200,100$, and 50). Hereinafter, a "PosCount" refers to a basket of positive class instances, and a "PosCount" of "all" specifically refers to all the original positive instances. 
Table 3 Medicare Part B average results

\begin{tabular}{llllllllll}
\hline PosCount & $\mathbf{5 0}$ & $\mathbf{1 0 0}$ & $\mathbf{2 0 0}$ & $\mathbf{3 0 0}$ & $\mathbf{4 0 0}$ & $\mathbf{5 0 0}$ & $\mathbf{7 5 0}$ & $\mathbf{1 0 0 0}$ & All \\
\hline a. AUC & & & & & & & & & \\
GBT & 0.6906 & 0.7344 & 0.7649 & 0.7822 & 0.7862 & 0.7914 & 0.7888 & 0.7957 & 0.7945 \\
LR & 0.7368 & 0.7477 & 0.7737 & 0.7779 & 0.7946 & 0.7973 & 0.8006 & 0.7998 & 0.8057 \\
RF & 0.6059 & 0.6375 & 0.7214 & 0.7214 & 0.7369 & 0.7503 & 0.7614 & 0.7800 & 0.7962 \\
b. GM & & & & & & & & & \\
GBT & 0.0063 & 0 & 0.0032 & 0.0052 & 0.0045 & 0.0020 & 0.0033 & 0.0042 & 0.0112 \\
LR & 0 & 0 & 0.0032 & 0.0077 & 0.0067 & 0.0080 & 0.0065 & 0.0156 & 0.0153 \\
RF & 0 & 0 & 0 & 0 & 0 & 0 & 0 & 0 & 0.0012 \\
c. AUPRC & & & & & & & & & \\
GBT & 0.0006 & 0.0003 & 0.0018 & 0.0020 & 0.0019 & 0.0027 & 0.0035 & 0.0046 & 0.0055 \\
LR & 0.0001 & 0.0002 & 0.0005 & 0.0013 & 0.0008 & 0.0017 & 0.0019 & 0.0025 & 0.0031 \\
RF & 0.0002 & 0.0001 & 0.0011 & 0.0010 & 0.0011 & 0.0013 & 0.0016 & 0.0025 & 0.0037 \\
\hline
\end{tabular}

Table 4 POSTSlowloris Combined average results

\begin{tabular}{llllllllll}
\hline PosCount & $\mathbf{5 0}$ & $\mathbf{1 0 0}$ & $\mathbf{2 0 0}$ & $\mathbf{3 0 0}$ & $\mathbf{4 0 0}$ & $\mathbf{5 0 0}$ & $\mathbf{7 5 0}$ & $\mathbf{1 0 0 0}$ & All \\
\hline $\begin{array}{lllllll}\text { a. AUC } \\
\text { GBT }\end{array}$ & & & & & & & & & \\
G948 & 0.9912 & 0.9963 & 0.9951 & 0.9903 & 0.9965 & 0.9979 & 0.9979 & 0.9990 \\
LR & 0.9903 & 0.9952 & 0.9915 & 0.9907 & 0.9841 & 0.9888 & 0.9909 & 0.9888 & 0.9877 \\
RF & 0.9809 & 0.9824 & 0.9818 & 0.9762 & 0.9764 & 0.9769 & 0.9766 & 0.9775 & 0.9753 \\
b. GM & & & & & & & & & \\
GBT & 0.6951 & 0.7422 & 0.7769 & 0.7723 & 0.7949 & 0.7745 & 0.7766 & 0.7484 & 0.7883 \\
LR & 0 & 0.0313 & 0.1861 & 0.4800 & 0.6661 & 0.7392 & 0.7384 & 0.7121 & 0.8031 \\
RF & 0 & 0 & 0 & 0 & 0 & 0 & 0 & 0 & 0 \\
c. AUPRC & & & & & & & & & \\
GBT & 0.5465 & 0.6266 & 0.6572 & 0.6887 & 0.7363 & 0.7202 & 0.7561 & 0.7605 & 0.9197 \\
LR & 0.0874 & 0.2199 & 0.3316 & 0.4235 & 0.4738 & 0.5134 & 0.5791 & 0.5842 & 0.7942 \\
RF & 0.0856 & 0.2166 & 0.2875 & 0.3551 & 0.4518 & 0.4444 & 0.4974 & 0.4957 & 0.6325 \\
\hline
\end{tabular}

Generally speaking, from Table 3, part a (AUC), we observe that the performance increases when the number of PosCounts increases for all three learners, and "all" positives reports the best performance. For parts b (GM), and c (AUPRC), we can see that the results are almost zero for all PosCounts. Particularly noticeable are the distinct 0 values for RF for all PosCounts in part b. This means that RF failed to correctly classify any positive counts, thus resulting in a $\mathrm{TP}_{\text {rate }}$ value of 0 . Consequently, the GM score, which relies on the product of $\mathrm{TP}_{\text {rate }}$ and $\mathrm{TN}_{\text {rate }}$, is also 0 . Note that RF with 100 trees builds 100 Decision Trees separately and then takes majority voting. Therefore, if in every instance prediction, the majority (more than 50) trees decided incorrectly, the final classification for RF will also be incorrect.

In Table 4, the results from part a (AUC) show very high-performance results for all three learners, no matter which PosCount is selected. One possible reason may be due to the fact that AUC values can mislead in some situations with severely imbalanced datasets. In part b, we see that GBT performed the best, on average, among all the PosCounts, while LR performed the best, on average, among all three learners. It is clear 
that RF performed the worst with its distinct 0 values. A reason for this behavior has already been discussed in the previous paragraph. Part $\mathrm{c}$ of the table shows the results for AUPRC. We observe, for the most part, that the performance increases when the number of PosCounts increases for all three learners, and "all" positives reports the best performance. Furthermore, GBT reports the best performance, followed by LR and RF. It is very noticeable that GBT performs reasonably well with even a PosCounts of 50, compared to LR and RF.

Table 5 shows the ANalysis Of VAriance (ANOVA) [38] results used to analyze the differences among group means. Each row within the table represents an ANOVA test for a specific combination of case study, learner, and performance metric. Results with

Table 5 One-way ANOVA

\begin{tabular}{|c|c|c|c|c|c|c|c|c|}
\hline Case study & Learner & Metrics & Term & df & sum-sq & mean-sq & F-value & $p$-value \\
\hline \multirow[t]{18}{*}{ Medicare Part B } & \multirow[t]{6}{*}{ GBT } & \multirow[t]{2}{*}{ AUC } & RarePos & 8 & 0.5039 & 0.0630 & \multirow[t]{2}{*}{40.0596} & \multirow[t]{2}{*}{$6.88 \times 10^{-48}$} \\
\hline & & & Residuals & 441 & 0.6933 & 0.0016 & & \\
\hline & & \multirow[t]{2}{*}{ AUPR } & RarePos & 8 & 0.0012 & $1.48 \times 10^{-4}$ & \multirow[t]{2}{*}{14.2490} & \multirow[t]{2}{*}{$1.61 \times 10^{-22}$} \\
\hline & & & Residuals & 441 & 0.0046 & $1.04 \times 10^{-5}$ & & \\
\hline & & \multirow[t]{2}{*}{ GM } & RarePos & 8 & 0.0039 & $4.92 \times 10^{-4}$ & \multirow[t]{2}{*}{0.8877} & \multirow[t]{2}{*}{0.5267} \\
\hline & & & Residuals & 441 & 0.2444 & $5.54 \times 10^{-4}$ & & \\
\hline & \multirow[t]{6}{*}{ LR } & \multirow[t]{2}{*}{ AUC } & RarePos & 8 & 0.2464 & 0.0308 & \multirow[t]{2}{*}{17.3412} & \multirow[t]{2}{*}{$1.69 \times 10^{-22}$} \\
\hline & & & Residuals & 441 & 0.7833 & 0.0018 & & \\
\hline & & \multirow[t]{2}{*}{ AUPR } & RarePos & 8 & $4.35 \times 10^{-4}$ & $5.44 \times 10^{-5}$ & \multirow[t]{2}{*}{17.5688} & \multirow[t]{2}{*}{$8.70 \times 10^{-23}$} \\
\hline & & & Residuals & 441 & 0.0014 & $3.10 \times 10^{-6}$ & & \\
\hline & & \multirow[t]{2}{*}{ GM } & RarePos & 8 & 0.0128 & 0.0016 & \multirow[t]{2}{*}{2.8390} & \multirow[t]{2}{*}{0.0044} \\
\hline & & & Residuals & 441 & 0.2490 & $5.65 \times 10^{-4}$ & & \\
\hline & \multirow[t]{6}{*}{$\mathrm{RF}$} & \multirow[t]{2}{*}{ AUC } & RarePos & 8 & 1.6019 & 0.2002 & \multirow[t]{2}{*}{99.2109} & \multirow[t]{2}{*}{$1.26 \times 10^{-93}$} \\
\hline & & & Residuals & 441 & 0.8901 & 0.0020 & & \\
\hline & & \multirow[t]{2}{*}{ AUPR } & RarePos & 8 & $5.07 \times 10^{-4}$ & $6.33 \times 10^{-5}$ & 18.2116 & $1.35 \times 10^{-23}$ \\
\hline & & & Residuals & 441 & 0.0015 & $3.48 \times 10^{-6}$ & & \\
\hline & & GM & RarePos & 8 & $6.30 \times 10^{-5}$ & $7.88 \times 10^{-6}$ & 1.0000 & 0.4352 \\
\hline & & & Residuals & 441 & 0.0035 & $7.88 \times 10^{-6}$ & & \\
\hline POSTSlowloris & GBT & AUC & RarePos & 8 & 0.0036 & $4.46 \times 10^{-4}$ & 5.0004 & $5.96 \times 10^{-6}$ \\
\hline Combined & & & Residuals & 441 & 0.0394 & $8.93 \times 10^{-5}$ & & \\
\hline & & AUPR & RarePos & 8 & 4.3164 & 0.5395 & 100.6363 & $1.69 \times 10^{-94}$ \\
\hline & & & Residuals & 441 & 2.3644 & 0.0054 & & \\
\hline & & GM & RarePos & 8 & 0.3755 & 0.0469 & 16.7467 & $9.59 \times 10^{-22}$ \\
\hline & & & Residuals & 441 & 1.2360 & 0.0028 & & \\
\hline & $L R$ & $A \cup C$ & RarePos & 8 & 0.0037 & $4.60 \times 10^{-4}$ & 3.8097 & $2.42 \times 10^{-4}$ \\
\hline & & & Residuals & 441 & 0.0533 & $1.21 \times 10^{-4}$ & & \\
\hline & & AUPR & RarePos & 8 & 17.8364 & 2.2296 & 684.9220 & $2.88 \times 10^{-243}$ \\
\hline & & & Residuals & 441 & 1.4355 & 0.0033 & & \\
\hline & & GM & RarePos & 8 & 42.2446 & 5.2806 & 481.2679 & $1.74 \times 10^{-212}$ \\
\hline & & & Residuals & 441 & 4.8387 & 0.0110 & & \\
\hline & RF & AUC & RarePos & 8 & 0.0029 & $3.67 \times 10^{-4}$ & 5.3050 & $2.28 \times 10^{-6}$ \\
\hline & & & Residuals & 441 & 0.0305 & $6.91 \times 10^{-5}$ & & \\
\hline & & AUPR & RarePos & 8 & 11.1250 & 1.3906 & 403.8733 & $1.37 \times 10^{-197}$ \\
\hline & & & Residuals & 441 & 1.5185 & 0.0034 & & \\
\hline & & GM & RarePos & 8 & 0 & 0 & $\mathrm{NaN}$ & $\mathrm{NaN}$ \\
\hline & & & Residuals & 441 & 0 & 0 & & \\
\hline
\end{tabular}


one factor, i.e. PosCounts, are shown to be significant at a $5 \%$ significance level for most rows. GM, for all three learners with the Medicare Part B dataset, shows insignificant levels between the PosCount group means. For RF and GM with the POSTSlowloris Combined case study, the ANOVA test failed to perform.

In order to evaluate which group factors are significant, a Tukey's Honestly Significant Difference (HSD) [39] test is performed to show the results for each PosCount (Table 6) and each learner (Table 7). Letter groups assigned via Tukey's HSD test indicate similarity or significant differences in results within each factor. As an example, the combination of GBT and AUC for the Medicare Part B case study results in an assignment of groups ' $\mathrm{d}$ ' and ' $\mathrm{c}$ ' to PosCounts of 50 and 100, respectively. Table 7 shows that GBT performed the best overall, being in group 'a' for all three metrics in the POSTSlowloris case study, and in group 'a' for the AUPRC and GM metrics in the Medicare Part B case study.

From Table 6, we observe that performance scores for the Medicare Part B case study become particularly poor at PosCounts of 200 and below, whereas for the POSTSlowloris

Table 6 Tukey's HSD Test (PosCounts)

\begin{tabular}{|c|c|c|c|c|c|c|c|c|c|c|}
\hline \multirow[t]{2}{*}{ Case study } & \multirow{2}{*}{$\begin{array}{l}\text { Learner } \\
\text { Sampling }\end{array}$} & \multicolumn{3}{|l|}{ GBT } & \multicolumn{3}{|l|}{ LR } & \multicolumn{3}{|l|}{ RF } \\
\hline & & AUC & AUPR & GM & AUC & AUPR & GM & AUC & AUPR & GM \\
\hline \multirow[t]{9}{*}{ a. Medicare Part B } & 50 & d & $d$ & - & e & e & - & $f$ & $d$ & - \\
\hline & 100 & $c$ & d & - & de & e & - & e & d & - \\
\hline & 200 & $b$ & $\mathrm{~cd}$ & - & $\mathrm{cd}$ & de & - & d & $\mathrm{cd}$ & - \\
\hline & 300 & $a b$ & $\mathrm{~cd}$ & - & $b c$ & $\mathrm{~cd}$ & - & $d$ & $\mathrm{~cd}$ & - \\
\hline & 400 & $a b$ & $\mathrm{~cd}$ & - & $a b c$ & cde & - & $\mathrm{cd}$ & $\mathrm{cd}$ & - \\
\hline & 500 & a & $b c$ & - & $a b c$ & $b c$ & - & $c$ & $\mathrm{~cd}$ & - \\
\hline & 750 & $a b$ & $a b c$ & - & $a b$ & bc & - & bc & bc & - \\
\hline & 1000 & a & $a b$ & - & $a b c$ & $a b$ & - & $a b$ & $b$ & - \\
\hline & All & a & $\mathrm{a}$ & - & a & $\mathrm{a}$ & - & a & a & - \\
\hline \multirow[t]{9}{*}{ b. POSTSlowloris Combined } & 50 & $a b c$ & $f$ & $d$ & $a b c$ & $\mathrm{~h}$ & $f$ & $a b c$ & g & - \\
\hline & 100 & bc & e & $C$ & a & g & $f$ & a & $f$ & - \\
\hline & 200 & $a b$ & de & $a b$ & $a b$ & $f$ & e & $a b$ & e & - \\
\hline & 300 & $a b c$ & $\mathrm{~cd}$ & $a b c$ & $a b c$ & e & $d$ & $\mathrm{~cd}$ & $d$ & - \\
\hline & 400 & c & $b$ & $\mathrm{a}$ & c & $d$ & $c$ & $\mathrm{~cd}$ & $c$ & - \\
\hline & 500 & $a b$ & $b c$ & $a b c$ & $a b c$ & $c$ & $a b$ & bcd & $c$ & - \\
\hline & 750 & $\mathrm{a}$ & $b$ & $a b$ & $a b$ & $b$ & $a b$ & $\mathrm{~cd}$ & $b$ & - \\
\hline & 1000 & $a$ & $b$ & $b c$ & $a b c$ & $b$ & $b c$ & abcd & $b$ & - \\
\hline & All & $a$ & $a$ & $\mathrm{a}$ & $b c$ & $a$ & $\mathrm{a}$ & $d$ & $a$ & - \\
\hline
\end{tabular}

Table 7 Tukey's HSD Test (Learners)

\begin{tabular}{lllll}
\hline Case study & Learner & Metrics & & \\
\cline { 4 - 5 } & & AUC & AUPRC & GM \\
\hline Medicare Part B & GBT & $\mathrm{b}$ & $\mathrm{a}$ & $\mathrm{a}$ \\
& LR & $\mathrm{a}$ & $\mathrm{b}$ & $\mathrm{a}$ \\
POSTSlowloris Combined & RF & $\mathrm{c}$ & $\mathrm{b}$ & $\mathrm{b}$ \\
& GBT & $\mathrm{a}$ & $\mathrm{a}$ & $\mathrm{a}$ \\
& LR & $\mathrm{b}$ & $\mathrm{b}$ & $\mathrm{b}$ \\
& RF & $\mathrm{c}$ & $\mathrm{c}$ & $\mathrm{c}$ \\
\hline
\end{tabular}


Combined case study, a noticeable deterioration of performance occurs at PosCounts of 100 and below. As a result, we infer that PosCounts of 200 and below, and 100 and below, are solid indicators of class rarity for the first and second case study, respectively.

\section{Conclusion}

We employ three learners (GBT, LR, RF) and three performance metrics (AUC, GM, AUPRC) to uniquely investigate class rarity in big data. Through our comparative analysis, we demonstrate the effectiveness and versatility of our method with two case studies involving imbalanced big data from different application domains.

For the Medicare Part B case study, we observe that classification performance scores for the learners generally improve for the AUC metric as the number of PosCounts increases, with "all" positives reporting the best performance. The other metrics have scores of zero or approximately zero. With regard to the POSTSlowloris Combined case study, the AUC metric yields very high performance results for the learners, while corresponding scores for the other metrics are, for the most part, noticeably lower. With the exception of the GM metric score obtained by the RF learner, scores for the learners generally improve with the AUPRC and GM metrics as the number of PosCounts increases.

Our results show that the GBT learner performs the best for both case studies. We also determined that PosCounts of 200 and below, and 100 and below, are strong indicators of class rarity for the first and second case study, respectively

Future work with big data and rarity will involve additional performance metrics, and also the investigation of data from other application domains.

\section{Abbreviations}

ANOVA: Analysis of variance; AUC: Area under the Roc Curve; AUPRC: Area under the precision-recall curve; CM: Confusion matrix; CV: Cross-validation; DDoS: Distributed denial of service; EUS: Evolutionary undersampling; FN: False negative; FP: False positive; FPrate: False positive rate; GBT: Gradient-boosted trees; GM: Geometric mean; HDFS: Hadoop distributed file system; HPC: High performance computing; HSD: Honestly significant difference; HTTP: Hypertext transfer protocol; LEIE: List of excluded individuals/entities; LR: Logistic regression; ML: Machine learning; MLlib: Machine learning library; NSF: National Science Foundation; PPV: Positive Predictive Value; RF: Random forest; ROC: Receiver Operating Characteristic; ROS: Random oversampling; RUS: Random undersampling; SMOTE: Synthetic Minority Over-sampling Technique; TN: True negative; TN rate: True Negative Rate; TP: True Positive; TP rate: True Positive Rate; YARN: Yet another resource negotiator.

\section{Acknowledgements}

We would like to thank the reviewers in the Data Mining and Machine Learning Laboratory at Florida Atlantic University. Additionally, we acknowledge partial support by the National Science Foundation (NSF) (CNS-1427536). Opinions, findings, conclusions, or recommendations in this paper are the authors' and do not reflect the views of the NSF.

\section{Authors' contributions}

TH conceived and designed the research, performed the implementation and experimentation, and performed the evaluation and validation. TH and JLL drafted the manuscript. JLL performed the primary literature review for this work. RAB prepared the Medicare dataset. All authors provided feedback to TH and helped shape the research. TMK introduced this topic to $\mathrm{TH}$, and helped to complete and finalize this work. All authors read and approved the final manuscript.

\section{Funding}

Not applicable.

Availability of data and materials

Not applicable.

Ethics approval and consent to participate

Not applicable.

Consent for publication

Not applicable. 


\section{Competing interests}

The authors declare that they have no competing interests.

Received: 9 December 2019 Accepted: 4 March 2020

Published online: 16 March 2020

\section{References}

1. Katal A, Wazid M, Goudar R. Big data: issues, challenges, tools and good practices. In: 2013 Sixth International Conference on contemporary computing (IC3). NewYork: IEEE; 2013. p. 404-409.

2. Leevy JL, Khoshgoftaar TM, Bauder RA, Seliya N. A survey on addressing high-class imbalance in big data. J Big Data. 2018;5(1):42.

3. Soltysik RC, Yarnold PR. Megaoda large sample and big data time trials: separating the chaff. Optim Data Anal. 2013;2:194-7.

4. Cao M, Chychyla R, Stewart T. Big data analytics in financial statement audits. Account Horizons. 2015;29(2):423-9.

5. Bauder RA, Khoshgoftaar TM, Hasanin, T. An empirical study on class rarity in big data. In: 2018 17th IEEE International Conference on machine learning and applications (ICMLA). Newyork: IEEE ; 2018. p. 785-790. IEEE

6. Bauder R, Khoshgoftaar T. Medicare fraud detection using random forest with class imbalanced big data. In: 2018 IEEE International Conference on information reuse and integration (IRI). Newyork: IEEE; 2018. p. 80-87.

7. Witten IH, Frank E, Hall MA, Pal CJ. Data mining: practical machine learning tools and techniques. Amsterdam: Morgan Kaufmann; 2016.

8. Olden JD, Lawler JJ, Poff NL. Machine learning methods without tears: a primer for ecologists. Q Rev Biol. 2008;83(2):171-93.

9. Galindo J, Tamayo P. Credit risk assessment using statistical and machine learning: basic methodology and risk modeling applications. Comput Econ. 2000;15(1):107-43.

10. Seliya N, Khoshgoftaar TM, Van Hulse J. A study on the relationships of classifier performance metrics. In: 200921 st IEEE International Conference on tools with artificial intelligence. Newyork: IEEE; 2009. p. 59-66.

11. Triguero I, Galar M, Merino D, Maillo J, Bustince H. Herrera, F. Evolutionary undersampling for extremely imbalanced big data classification under apache spark. In: Evolutionary Computation (CEC), 2016 IEEE Congress on; Newyork: IEE; 2016. p. 640-647.

12. Apache Hadoop. http://hadoop.apache.org/

13. Venner J. Pro Hadoop. Berkeley: Apress; 2009

14. White T. Hadoop: the definitive guide. Sebastopol: O'Reilly Media Inc; 2012

15. Bauder RA, Khoshgoftaar TM, Hasanin T. Data sampling approaches with severely imbalanced big data for medicare fraud detection. In: 2018 IEEE 30th International Conference on tools with artificial intelligence (ICTAI). Newyork: IEEE; 2018. p. 137-142.

16. Johnson JM, Khoshgoftaar TM. Medicare fraud detection using neural networks. J Big Data. 2019:6(1):63.

17. Calvert C, Khoshgoftaar TM, Kemp C, Najafabadi MM. Detecting slow http post dos attacks using netflow features. In: The Thirty-second International FLAIRS Conference (2019).

18. Calvert C, Khoshgoftaar TM, Kemp C, Najafabadi MM. Detection of slowloris attacks using netflow traffic. In: 24th ISSAT International Conference on reliability and quality in design. 2018; p. 191-196.

19. García S, Herrera F. Evolutionary undersampling for classification with imbalanced datasets: proposals and tax onomy. Evol Comput. 2009;17(3):275-306.

20. Del Río S, López V, Benítez JM, Herrera F. On the use of mapreduce for imbalanced big data using random forest. Inf Sci. 2014;285:112-37.

21. Baughman AK, Chuang W, Dixon KR, Benz Z, Basilico J. Deepqa jeopardy! gamification: a machine-learning perspective. IEEE Trans Comput Intell Al Games. 2013;6(1):55-66.

22. Ferrucci D, Brown E, Chu-Carroll J, Fan J, Gondek D, Kalyanpur AA, Lally A, Murdock JW, Nyberg E, Prager J, et al. Building watson: an overview of the deepqa project. Al Mag. 2010;31(3):59-79.

23. LEIE: Medicare provider utilization and payment data: physician and other supplier. https://oig.hhs.gov/exclusions/ index.asp

24. Liu Y-h, Zhang H-q, Yang Y-j. A dos attack situation assessment method based on qos. In: Proceedings of 2011 International Conference on computer science and network technology. Newyork: IEEE; 2011. p. 1041-1045.

25. Yevsieieva O, Helalat SM. Analysis of the impact of the slow http dos and ddos attacks on the cloud environment. In: 2017 4th International scientific-practical Conference problems of infocommunications. Science and technology (PIC S\&T). Newyork: IEEE; 2017. p. 519-523.

26. Hirakaw T, Ogura K, Bista BB, Takata T. A defense method against distributed slow http dos attack. In: 2016 19th International Conference on network-based information systems (NBiS)). Newyork: IEEE; 2016. p. 519-523.

27. Slowloris.py. https://github.com/gkbrk/slowloris

28. Apache Spark MLlib. https://spark.apache.org/mllib/

29. Zaharia M, Chowdhury M, Franklin MJ, Shenker S, Stoica I. Spark: cluster computing with working sets. HotCloud. 2010;10:95.

30. Meng X, Bradley J, Yuvaz B, Sparks E, Venkataraman S, Liu D, Freeman J, Tsai D, Amde M, Owen S, et al. Mllib: Machine learning in apache spark. JMLR. 2016;17(34):1-7.

31. Vavilapalli VK, Murthy AC, Douglas C, Agarwal S, Konar M, Evans R, Graves T, Lowe J, Shah H, Seth S, et al. Apache hadoop yarn: yet another resource negotiator. In: Proceedings of the 4th Annual Symposium on cloud computing. Newyork: ACM; 2013. p. 5.

32. Herland M, Khoshgoftaar TM, Bauder RA. Big data fraud detection using multiple medicare data sources. J Big Data. 2018;5(1):29. 
33. Seiffert C, Khoshgoftaar TM, Van Hulse J, Napolitano A. Mining data with rare events: a case study. In: 19th IEEE International Conference on tools with artificial intelligence (ICTAI 2007). Newyork: IEEE; 2007. vol 2, p. 132-139. IEEE.

34. Herland M, Khoshgoftaar TM, Wald R. A review of data mining using big data in health informatics. J Big data. 2014;1(1):2

35. Saito T, Rehmsmeier M. The precision-recall plot is more informative than the roc plot when evaluating binary classifiers on imbalanced datasets. PLoS ONE. 2015;10:0118432.

36. Kohavi R. A study of cross-validation and bootstrap for accuracy estimation and model selection. In: Proceedings of the 14th International Joint Conference on artificial intelligence. Burlington: Morgan Kaufmann Publishers Inc; 1995. Vol 2, p. 1137-1143.

37. Van Hulse J, Khoshgoftaar TM, Napolitano A. An empirical comparison of repetitive undersampling techniques. In: 2009 IEEE International Conference on information reuse \& integration. Newyork: IEEE; 2009. p. 29-34.

38. Gelman A. Analysis of variance-why it is more important than ever1. Ann Stat. 2005;33(1):1-53.

39. Tukey JW. Comparing individual means in the analysis of variance. Biometrics. 1949;1:99-114.

\section{Publisher's Note}

Springer Nature remains neutral with regard to jurisdictional claims in published maps and institutional affiliations.

\section{Submit your manuscript to a SpringerOpen ${ }^{\circ}$} journal and benefit from:

- Convenient online submission

- Rigorous peer review

Open access: articles freely available online

- High visibility within the field

- Retaining the copyright to your article

Submit your next manuscript at $\boldsymbol{\Delta}$ springeropen.com 